INRA Prod. Anim., 2008, 21 (1), 137-144

\title{
Quel avenir pour les recherches en productions et santé animales?
}

P. HERPIN, B. CHARLEY

INRA, Collège de Direction, 147 rue de l'Université, F-75338 Paris, France

Courriel :Patrick.Herpin@rennes.inra.fr

Les recherches en productions animales de demain devront tout à la fois produire des données génériques pour la connaissance du vivant et proposer des systèmes de production innovants et compétitifs, en rupture avec l'existant. Elles devront prendre en compte la dimension mondiale des problématiques et s'accompagner d'une large ouverture disciplinaire et partenariale.

\section{1 / Contexte et enjeux}

Après avoir été l'un des acteurs majeurs de la modernisation de l'élevage après guerre, la recherche agronomique des 20 dernières années a largement contribué à l'essor du secteur agroalimentaire français, en intégrant les innovations provenant des avancées très importantes dans le domaine des sciences du vivant (biologie cellulaire, génétique moléculaire, sciences des comportements). Les quelques exemples de recherche et d'innovation rassemblés dans ce numéro spécial de la revue INRA Productions Animales montrent combien les progrès ont été spectaculaires, les approches diverses, les innovations nombreuses dans le domaine de l'animal et des produits animaux. Aujourd'hui, deux grands enjeux sont venus replacer l'agronomie et les productions animales au cœur des débats de société : la prise de conscience de la nécessité de se situer dans une perspective de développement durable ${ }^{1}$ pour conserver et transmettre notre patrimoine environnemental aux futures générations et la mondialisation de l'économie qui impose une concurrence exacerbée entre grandes régions de production.

\section{1 / Développement durable}

Le développement durable est devenu un objectif incontesté, même si la manière d'y parvenir fait encore débat et s'il reste difficile de poser les bonnes questions de recherche dans ce domaine. Il nécessite une mobilisation massive et coordonnée des sciences biotech- niques et sociales (Boiffin et al 2004). Il réaffirme les impératifs de production et de compétitivité économique des élevages et des entreprises agroalimentaires, première condition de la durabilité, mais simultanément et avec un poids au moins égal à celui des critères économiques classiques, il met en avant les dimensions sociales et environnementales. Les chercheurs en productions animales doivent donc maintenant tout faire pour que les pratiques d'élevage actuelles évoluent fortement, deviennent moins négatives vis-à-vis de l'environnement, plus recevables par le consommateur et le citoyen, notamment en matière de bien-être animal et de sécurité alimentaire, et aboutissent à des produits mieux adaptés aux besoins nutritionnels et à la santé de l'Homme. Dans la plupart des cas, ce sont désormais des recherches interdisciplinaires et systémiques, soutenues par un partenariat élargi et une coordination étroite avec les acteurs des filières, qui permettront d'apporter des réponses pertinentes à ces questions, cruciales pour l'avenir des productions animales.

\section{2 / Mondialisation des problé- matiques liées aux productions} animales

La dimension mondiale des problématiques abordées en sciences animales est de plus en plus présente (changement climatique, gestion de la biodiversité, qualité de l'eau, qualité et sécurité de l'alimentation, maladies émergentes, bioénergies). Cette dimension est imposée par la mondialisation des échanges voulue par la communau- té internationale, mais elle s'impose également du fait de la nature même des questions posées aux productions animales qui tournent toutes autour de l'insertion des systèmes d'élevage actuels dans leur environnement. Le climat, la biodiversité, l'eau et l'air, pas plus que les pathogènes n'ont de frontières. De ce fait, elle place bien souvent les pratiques d'élevage, les animaux ou leurs produits au cœur de débats de société et de polémiques qui ne pourront trouver une issue que si l'on est capable de produire de nouvelles connaissances sur la maîtrise du vivant, de renouveler nos pratiques et de mieux gérer les risques qu'elles peuvent générer. Cette mondialisation doit nourrir nos réflexions et nos programmes.

\section{3 / Nourrir le monde tout en préservant la santé humaine et l'environnement}

Dans ce cadre ainsi élargi, le défi principal proposé à l'agriculture et à l'élevage reste encore de nourrir le monde, la situation d'autosuffisance alimentaire ne concernant aujourd'hui qu'une faible part de l'humanité. Sur un total de 6,5 milliards d'habitants, 2 milliards sont mal nourris et 850 millions souffrent de la faim car disposant de moins de 2200 calories par jour (Prospective «AgriMonde» à paraître). On peut alors estimer qu'en 2050, l'augmentation de la population mondiale à 9 milliards d'habitants s'accompagnera d'un doublement de la demande en protéines animales. Cette forte augmentation mondiale sera essentiellement le fait des pays les plus

${ }^{1}$ Défini ici comme le développement qui permet de satisfaire les besoins de la génération actuelle, sans entamer ceux des générations à venir. 
pauvres et des pays émergents (en relation avec la hausse des revenus et des densités de population) alors que la consommation aura tendance à se réduire dans les pays développés, notamment en Europe de l'ouest. En France, ce repli est déjà manifeste et probablement durable, les ménages de moins de 35 ans achetant en moyenne deux fois moins de viande que leurs aînés de plus de 50 ans (Devine 2003). Aux questions du Sud et des échanges agricoles s'ajouteront alors celles de notre contribution directe ou indirecte à cette fourniture accrue de protéines animales qui impactera le volume et la densité de nos élevages métropolitains. Cette évolution de la demande alimentaire à l'échelle de la planète est d'autant plus complexe qu'à la croissance démographique se surajoutera la place croissante donnée aux cultures à vocations non alimentaires.

Il faut et il faudra aussi composer avec la nécessité de fournir des produits conformes aux exigences de sécurité sanitaire et aux besoins nutritionnels des différentes catégories et tranches d'âge des populations. Garantir au consommateur des produits sains, accessibles et adaptés à une alimentation équilibrée devient une priorité. La qualité de l'alimentation est en effet devenue en quelques années un sujet de société au cœur d'enjeux socio-économiques considérables. La place des produits animaux y est de plus en plus soumise à interrogations et leur image souvent dégradée, notamment vis-à-vis de considérations nutritionnelles. Les relations entre l'élevage, les produits animaux et la santé humaine vont en effet bien au-delà de l'apport de protéines et d'oligo-éléments puisque d'une part, les produits animaux sont accusés de contribuer au développement des troubles de santé liés aux diètes trop riches (obésité, diabète, maladies cardiovasculaires...), et d'autre part, l'élevage et les animaux d'élevage jouent un rôle important dans l'apparition ou la propagation de nombreuses maladies émergentes et des zoonoses (toxoplasmose, grippe aviaire, salmonelloses, EST, mycobactériose...). Mais les produits animaux apportent également des éléments nutritionnels nécessaires au bon fonctionnement de l'organisme (vit B12...) et l'appréhension globale de «l'effet moyen» des produits issus de l'élevage n'est donc pas aisée. Une réflexion approfondie sera nécessaire pour adapter notre dispositif de recherche à ce nouveau contexte sociétal.
La difficulté n'est pas tant de répondre à ces deux premiers défis mais également d'y ajouter une troisième exigence, tout aussi cruciale, la préservation de l'environnement et des ressources naturelles. L'autosuffisance alimentaire des pays développés a été atteinte au prix d'effets collatéraux sur l'environnement (qualité de l'eau, pesticides, biodiversité hébergée) et sur la structure des paysages et des territoires. Les impacts environnementaux de l'élevage ne doivent pas être minimisés et leur réduction devra faire l'objet d'efforts conséquents de recherche, avec des méthodes et un partenariat renouvelés, dans le contexte plus large du changement global. Un récent rapport (FAO 2006) a tenté de caractériser et de quantifier ces impacts de l'élevage (réchauffement climatique, biodiversité, ressources et qualité de l'eau, usage des terres et dégradation des sols...). Il met en avant l'utilisation massive des terres pour l'élevage : 3,4 milliards d'ha de pâtures, soit $26 \%$ des terres émergées, et 470 millions d'ha de terres dédiées à la production d'aliments du bétail, soit 33\% des terres arables. L'élevage représente $8 \%$ de l'ensemble de l'usage anthropique de l'eau, dont $7 \%$ pour la production d'aliments. De plus, si le développement de l'usage non-alimentaire de la production de biomasse crée de nouvelles finalités pour les productions agricoles, il questionne aussi fortement l'élevage puisqu'il augmentera la tension qui existe déjà sur les usages de l'eau et du sol, accentuera la déforestation (recherche de nouvelles surfaces cultivables) et posera la question de l'utilisation des sous-produits. Ce rapport FAO conclut que, directement ou indirectement, l'activité d'élevage contribue pour $18 \%$ aux émissions de gaz à effet de serre (35\% liés à la déforestation, $31 \%$ aux lisiers et fumiers ; $25 \%$ à la fermentation entérique, $7 \%$ à la production d'aliments) et que $20 \%$ des terres de parcours sont dégradées. Le constat n'est pas brillant non plus en termes de perte de biodiversité, de destruction/ dégradation des habitats (déforestation, pollution, désertification) ou d'exploitation de ressources marines pour la production de farines de poisson pour l'aquaculture. A noter cependant que la situation est très contrastée au plan mondial, le Brésil étant par exemple à lui seul responsable de $60 \%$ des émissions de gaz à effet de serre des activités liées à l'élevage. Mais il convient de moduler ce constat, en portant au crédit de l'élevage des ruminants l'entretien des prairies, l'occupation des territoires par l'Homme ou l'entretien des zones difficiles. Un récent rapport (Soussana et al 2007), issu du projet européen GREENGASS, met ainsi en évidence un bilan global équilibré de la production de gaz à effet de serre $\left(\mathrm{CO}_{2}, \mathrm{~N}_{2} \mathrm{O}\right.$, $\mathrm{CH}_{4}$ ) au niveau des prairies exploitées pour l'élevage des ruminants en Europe.

\section{4 / Ethique, expérimentation animale et élevage}

A ce constat mondial, il faut ajouter un dernier élément plus présent dans nos sociétés occidentales où le citoyen est de plus en plus éloigné des activités d'élevage, celui de l'évolution du statut de l'animal. Il pose la question de notre rapport à l'animal et retentit doublement sur la recherche agronomique à travers les techniques d'élevage que nous diffusons, mais également en relation avec nos activités expérimentales proprement dites. La recherche scientifique soulève, en effet, des questions éthiques de par l'utilisation des animaux dans ses protocoles expérimentaux. La quête du savoir justifie-t-elle d'effectuer des expériences qui sont susceptibles de causer la douleur et/ou la détresse des animaux ? Quelles sont les limites acceptables de l'expérimentation animale ? Quel objectif, quelles applications et quelle acceptabilité des travaux sur les biotechnologies dans le domaine de la génomique, de la physiologie et de la santé ? Les animaux d'élevage sont-ils bien traités? Nous devons éclairer ces débats par nos travaux et construire un dialogue confiant avec la société. Les campagnes d'opinion et la sensibilité du public envers les animaux, ainsi que la place grandissante du bien-être animal dans la réglementation et dans les modalités d'élevage soulignent l'importance d'approfondir les connaissances sur le sujet.

\section{2 / Démarches prospectives en cours}

Anticiper les évolutions futures et les prochaines questions de recherche est un exercice difficile. Avant d'esquisser quelques pistes et de pointer quelques priorités pour les recherches en productions animales et produits animaux à l'INRA, il convient de rappeler que de multiples démarches prospectives ont été réalisées ces dernières années (prospective génétique, prospective élevage de la DATAR) ou sont en cours. 
Trois prospectives ont été lancées récemment par l'INRA. "Agriculture 2013», avec le Crédit Agricole et Groupama, a pour objectif d'analyser les conséquences d'hypothèses d'évolution du contexte mondial, de la PAC, des stratégies des acteurs, sur l'agriculture et l'agroalimentaire français à l'horizon 2013. Cette date verra en effet une évolution marquée de la PAC, avec une réduction et une réorientation des aides. Cette analyse est d'autant plus nécessaire et urgente que l'examen du budget de l'Union Européenne de l'après 2013 est prévu en 2009, juste après le bilan de santé de la PAC prévu en 2008. Cette prospective met en exergue la grande vulnérabilité des productions animales françaises, notamment des herbivores, quels que soient les scénarios envisagés (Guyomard et al 2007). La prospective «AgriMonde» (agricultures et alimentations du monde en 2035) avec le CIRAD, a pour objectif de construire des scénarios d'évolution des productions, des consommations et des échanges agricoles mondiaux et d'en tirer les implications pour la recherche agronomique, les politiques publiques et les régulations internationales, en s'attachant aux finalités de sécurisation alimentaire et de développement durable. Enfin, «Nouvelles ruralités à l'horizon 2030» s'est donné comme ambition d'explorer les futurs possibles du monde rural. Ces trois prospectives devraient nous fournir dans un proche avenir des bases solides et des scénarios s'appuyant sur des modélisations quantitatives et originales pour nous permettre, avec un recul suffisant, de repenser les questions de recherche et de faire émerger de nouveaux programmes.

Du côté des filières animales, des réflexions prospectives sont aussi en cours. Citons par exemple la prospective INRA-IFREMER sur la pisciculture française à l'horizon 2021 qui vient de rendre ses conclusions : stagnation des volumes issus de la pêche, nécessité de protéger les ressources marines, changement climatique, demande accrue en produits aquatiques et évolution des habitudes alimentaires sont les grandes tendances qui nourrissent les scénarios proposés pour parvenir ou non à un développement durable de l'aquaculture. Dans le domaine avicole, l'INRA lance aussi avec l'ITAVI et de nombreux partenaires une prospective sur la filière avicole française à l'horizon 2020 , une filière très intégrée dont les systèmes de production sont largement dépendants des évolutions de marchés et des stratégies des opérateurs d'aval, et très en prise avec les grands enjeux évoqués plus haut dans ce document (bien-être et santé animale, impacts environnementaux, concurrence internationale).

\section{3 / Quelques orientations prioritaires}

Au carrefour de ces contextes socioéconomiques et scientifiques en évolution, quelques grands axes prioritaires de recherche sur l'animal, l'élevage et les produits animaux peuvent être tracés. Ils entrent totalement dans l'objectif d'une recherche agronomique finalisée pour une alimentation adaptée, un environnement préservé et une agriculture compétitive et durable, en lien avec l'ensemble des acteurs publics et privés. Ils se fondent sur les constats suivants :

- il est indispensable de soutenir conjointement l'acquisition de connaissances de base sur la biologie et la santé des organismes animaux et le développement d'approches systémiques intégratives et interdisciplinaires ; ces deux dimensions fonderont la biologie et l'agronomie de demain ;

- les chercheurs en sciences animales ont déployé de gros efforts ces dernières années pour s'approprier les concepts, les méthodes et les outils de la génomique (expression des gènes) et de la post-génomique (rôle des protéines dans les fonctions d'intérêt). Ces efforts doivent être poursuivis et intensifiés pour tirer un maximum de profit des multiples données accumulées et déboucher sur des innovations transférables et acceptables par la société ;

- un effort particulier de réflexion, de reformulation des questions de recherche et de structuration des partenariats doit être réalisé sur deux domaines de recherche qui méritent une attention particulière : l'élaboration des produits animaux (incluant leur perception par la société et leur part dans l'alimentation de l'Homme), et les relations élevage-environnement (impacts, aménités, contribution au changement global) qui méritent une approche pro-active, élargie et multipartenaires ;

- la conception et l'évaluation de nouveaux systèmes de production, intégrant les différentes dimensions du développement durable est une nécessité. Cela passe d'une part par des travaux associant expérimentation et modélisation, et d'autre part par le développement de démarches d'évaluation multicritères des systèmes élevages existants ou à développer ;
- la pathologie des différentes espèces d'animaux de rente, qui affecte fortement la rentabilité des élevages jusqu'à annihiler des années de progrès génétique, reste dominée par les processus infectieux et il faut poursuivre les efforts de recherches et de programmation sur les maladies infectieuses, zoonotiques et/ou émergentes, et le développement de méthodes de contrôle de ces maladies, en développant des liens avec le monde médical et les partenaires industriels (vaccins, médicaments) ;

- en combinant les outils génétiques et génomiques disponibles, la sélection doit s'orienter vers un objectif équilibré associant productivité, qualité des produits, robustesse des animaux et adaptation aux systèmes de production du futur ;

- l'INRA doit participer au dialogue science-société en éclairant le débat sur l'éthique par ses travaux sur les biotechnologies et le bien-être animal.

Pour s'approprier pleinement ces différentes dimensions, les chercheurs en sciences animales devront résolument ouvrir leurs réflexions et leurs projets à d'autres disciplines (écologie, agronomie, nutrition humaine, sociologie, économie de l'élevage et des produits...) et construire leurs questions de recherche en diversifiant leurs partenariats.

\section{1 / De la génomique à la post- génomique}

Le programme AGENAE (Analyse du génome des animaux d'élevage) relayé par le programme GENANIMAL et maintenant GENOMIQUE de l'ANR (Agence Nationale de la Recherche) a commencé à porter ses fruits. A court terme, les priorités scientifiques de ce programme seront :

- de renforcer les recherches sur les mécanismes de régulation de l'expression des gènes (variabilité génétique, empreinte, épigénétique, contrôle de la traduction) ;

- de prévoir la transposition des acquis de la recherche obtenus dans les espèces majeures aux productions dites «secondaires» (caprins, ovins, dinde, pintade, canard, poissons autres que salmonidés) dont le génome n'est pas connu (cartographie, approches comparatives) ;

- de construire en interne (exploitation du potentiel de nos unités expérimentales notamment) et en externe (avec les filières), le phénotypage à haut débit, qui va devenir le goulot 
d'étranglement des recherches en génomique (quels critères, quels modes d'enregistrement, quelle utilisation, quelle ouverture ?) ;

- d'organiser le génotypage à haut et moyen débit.

Couplée aux avancées dans le domaine de la bioinformatique et de la modélisation, cette dynamique ouvrira des perspectives ambitieuses d'intégration des connaissances. Dans ce contexte, nous soutenons la reconduction du GIS AGENAE avec le souci d'élargir et de conforter les partenariats, d'irriguer davantage de nouvelles thématiques (nutrition, interactions hôtes agents pathogènes ou xénobiotiques, métagénomique), d'exploiter les données issues du séquençage des génomes, et de faire évoluer le programme GENANIMAL/GENOMIQUE vers davantage de biologie intégrative et comparative (phylogénomique). Cette connaissance approfondie des génomes aura des implications considérables en matière de sélection, la sélection génomique, qu'il conviendra d'explorer, d'anticiper et de diffuser.

Il est aussi attendu des génomiciens animaux qu'ils s'approprient les nouveaux enjeux du développement durable et investissent notamment le domaine des relations entre élevage et environnement, tel qu'il est présenté dans le chapitre suivant (respect de l'environnement, adaptation au changement climatique, robustesse et adaptabilité des animaux...).

\section{2 / L'élevage : un acteur incontournable de l'environ- nement}

L'élevage est clairement associé, dans certains systèmes, à la dégradation de l'environnement et ses impacts négatifs risquent encore de s'aggraver si l'on tente de répondre à la demande mondiale croissante en protéines animales sans faire évoluer notre manière de produire. Les préoccupations sur les impacts environnementaux de l'élevage sont fondées au niveau planétaire et le monde de l'élevage doit résolument s'engager sur la problématique du développement durable. Grâce aux premiers travaux engagés, une partie des solutions techniques existe déjà pour réduire les émissions de méthane et/ou de nitrates (via l'alimentation, via le traitement des effluents, via la gestion de la fertilisation), pour gérer les ressources en eau (améliorer l'efficacité de l'irrigation, mieux gérer les effluents, mieux gérer les pâturages), pour maintenir la biodiversité (réduire la pression et gérer les interactions, développer une agriculture intégrée, adopter de bonnes pratiques agricoles, approches écologiques) ou pour maîtriser l'usage raisonné des anti-infectieux ou des anti-parasitaires. Il faut poursuivre dans ce sens et aller plus loin en intégrant aussi la production d'aménités environnementales par l'élevage (contribution à la biodiversité, séquestration de carbone, production d'énergie ou de fertilisants à partir des effluents, valorisation de coproduits issus des filières bioénergétiques, entretien des territoires...), les interrogations sur la production et l'utilisation de l'énergie, et en tentant de réconcilier élevage et écologie pour promouvoir une «agronomie intégrale» (Chevassusau-Louis 2006). Réciproquement, il nous faut analyser comment l'élevage pourra s'adapter au nouveau contexte du changement climatique (raréfaction de la ressource en eau, modifications de la flore prairiale, diffusion de nouveaux vecteurs arthropodes de maladies animales, élévation de la température de l'eau et de l'air...). Tout cela nécessite probablement une «remise à plat» des façons de produire et les biologistes animaux devront, plus que par le passé, jouer un rôle moteur et central dans les discussions et travaux à venir pour engager les ruptures nécessaires.

Pour y parvenir il convient, sans négliger les recherches disciplinaires, de privilégier les démarches interdisciplinaires, globales et systémiques, et de consolider les partenariats sur ce thème à la fois au sein des différents secteurs de l'INRA et avec les filières. Toutes les filières animales et certaines filières végétales doivent $\mathrm{y}$ travailler ensemble. Des initiatives de structuration sont en cours sous la forme d'un Réseau Mixte Technologique «Elevage-Environnement» associant recherche, développement et formation. Il faudra les prolonger, les consolider avec les démarches des chercheurs pour tenter de construire un grand programme partenarial, sur les relations entre élevage et environnement. L'opportunité est grande aussi sur ce dossier de construire une programmation et une réflexion commune avec d'autres organismes pour partager les acquis et les interrogations sur les problématiques du Nord comme du Sud.

Les problématiques concernent notamment : 1 / la recherche des indicateurs les plus pertinents pour la quantification et la modélisation des impacts des pratiques d'élevage, des flux des molécules en cause, des transferts de pollution, des fonctionnements prairiaux, de l'impact des micropolluants, 2/ les moyens de maîtrise des impacts, $3 /$ la conception intégrée de systèmes d'élevage écologiquement performants, 4/ les conséquences pour l'organisation des filières (intégration, relocalisation), 5/ l'adaptation de l'élevage au changement climatique. En termes de systèmes d'élevage, une réflexion spécifique est aussi nécessaire sur les notions d'intensif/extensif, à la fois en termes d'efficacité des productions et d'impact environnemental, social et écologique. L'intensification des productions préconisée par le rapport FAO s'applique par exemple avant tout à des systèmes actuellement très extensifs et très peu productifs de type Africain, et non pas aux élevages extensifs d'herbivores français. Inversement, il est clair qu'une augmentation de l'efficacité des productions (par l'alimentation, le système d'élevage ou la génétique) peut s'accompagner d'une réduction des rejets, des effectifs d'animaux et des surfaces nécessaires pour un objectif de production donné. Cela pose donc les questions du niveau d'approche et des indicateurs utilisés. L'efficacité de l'usage des ressources pour la production animale doit être pensée non seulement en termes de performance économique, mais aussi de performance environnementale et sanitaire.

\section{3 / Les produits animaux : quelle place dans l'alimentation de l'Homme demain ?}

On ne peut construire durablement nos travaux de recherche sans tenter, au préalable, de répondre à cette question. Le contexte général, rappelé en introduction, est paradoxal : une forte augmentation de la demande mondiale en protéines animales, de multiples mises en garde contre les risques associés à une consommation excessive de produits animaux, une progression des considérations éthiques ou philosophiques sur l'exploitation de l'animal par l'Homme. Avant d'aller plus loin, il convient donc de mener une réflexion large et prospective sur l'évolution de la place des produits animaux dans l'alimentation de l'homme, en prenant en compte l'ensemble des enjeux qui lui sont liés, qu'ils soient de nature économique, sociologique, nutritionnelle, zootechnique, environnementale ou sanitaire. Cette réflexion nous conduira à revisiter éventuellement nos programmes, priorités et dispositifs, à promouvoir de nouveaux projets et de nou- 
velles synergies, à nous interroger sur la manière de produire. La construction de la qualité nutritionnelle et organoleptique des produits animaux, au sein d'un régime alimentaire équilibré, leur qualité sanitaire en relation avec l'émergence de nouveaux modes d'élevage moins confinés devront faire l'objet d'une attention particulière, tout comme le transfert de xénobiotiques et de micropolluants au sein de la chaîne alimentaire. Cette notion de chaîne alimentaire devra être déclinée dans des programmes collaboratifs interdisciplinaires, la qualité des produits se construisant dès l'amont, au sein des systèmes d'élevage.

Les efforts d'acquisition de connaissances sur les caractéristiques des produits animaux, d'élimination de composés indésirables, méritent d'être poursuivis et accompagnés de travaux visant à évaluer les bénéfices/risques de leur consommation pour le fonctionnement et la santé de l'organisme humain. Les enjeux sont très importants en terme de communication et de transfert sur les caractéristiques nutritionnelles, sensorielles et sanitaires propres des produits animaux et les risques d'une consommation excessive ou carencée. Mais nos démarches de recherche devront être plus globales et plus intégratives, pour prendre en compte l'équilibre global du régime alimentaire. A cet égard, les effets associatifs et les complémentarités des apports nutritionnels animaux et végétaux mériteront une attention particulière et la définition de programmes de recherche communs avec les nutritionnistes et les spécialistes des produits végétaux.

A l'interface entre les deux thèmes précédents, les relations entre alimentation et environnement s'affichent de plus en plus comme l'un des axes de l'application du concept de développement durable : peut-on favoriser une production d'aliments qui associe santé de l'Homme et respect de l'environnement ? Décrire les liens complexes entre alimentation et environnement est un champ de recherche relativement récent et la production d'indicateurs fiables de type écobilans et analyse de cycle de vie (de la production à la consommation) reste délicate. Il n'en reste pas moins que «la manière de produire» est au cœur de cette problématique qui doit nous interpeller. L'origine et le mode de production constituent d'ailleurs des facteurs déterminants pour les économies d'énergie dans les systèmes de production des aliments
(Redlingshöfer 2006), et dans le cas de la viande, le module «élevage» domine largement les autres (transport, transformation...) en termes d'impact environnemental dans les écobilans.

\section{4 / L'animal : un statut en profonde évolution}

La mutation extraordinaire des systèmes de production animale et des filières au cours des 4 à 5 dernières décennies n'a pas suffisamment été expliquée aux consommateurs qui, dans le même temps, ont perdu leurs racines rurales et ont maintenant pour référence l'animal de compagnie. En parallèle, la progression des connaissances en éthologie et en psychologie cognitive grâce aux investissements des scientifiques des productions animales ont construit les notions de bien-être animal et de bientraitance, et ont commencé à faire évoluer, à juste titre, certains de nos systèmes de production pour lesquels le bien-être animal n'était auparavant qu'une variable d'ajustement. Par ailleurs, le recours à l'expérimentation animale est de plus en plus une source d'interrogations justifiées en matière d'éthique, de bien-traitance, voire même de relations homme-animal. Tous ces facteurs qui concourent à l'évolution du statut de l'animal d'élevage dans la société, interfèrent à la fois avec l'expérimentation animale, les activités d'élevage et l'acceptabilité des produits animaux. Ils doivent nous inciter à construire des réponses éclairantes permettant $1 /$ d'élaborer les systèmes d'élevage de demain, 2/ de faire évoluer et de rendre acceptables les innovations en biotechnologies animales et $3 /$ de construire notre dialogue avec la société.

Au carrefour de considérations socioculturelles, biologiques, zootechniques et morales, la définition du bien-être animal est d'ailleurs loin d'être unanimement partagée par les différentes communautés de pensée (Veissier et al 2007). Cette dimension continuera à être revisitée, enrichie, explicitée, notamment par la mise en œuvre d'approches pluridisciplinaires et d'une évaluation globale, incluant l'état de santé de l'animal. Elle sera prise en compte dans l'élaboration des systèmes d'élevage, sans toutefois tomber dans l'excès et sans perdre de vue que l'amélioration du bien-être animal doit remettre l'éleveur au cœur du système de production. C'est en effet lui qui est le mieux à même de comprendre ses animaux et de déceler leurs problèmes. Il faudra donc contribuer à l'acquisition de connaissances nouvelles, construire davantage nos questions de recherche en partenariat, contribuer à l'amélioration du dialogue science-société, et consolider notre rôle d'expert auprès des autorités compétentes.

Les techniques de clonage et de transgenèse animale sont porteuses de progrès scientifique et d'innovations considérables mais suscitent déjà des interrogations légitimes sur leur utilité et leur danger potentiel : quelle utilité dans les secteurs de l'agronomie, de la production industrielle, de la santé humaine ? Quelles contraintes éthiques (bien-être animal, responsabilité par rapport à des applications qui seraient détournées vers l'Homme...), pèsent sur le choix des transgènes et des modalités expérimentales et technologiques ? Quels risques sanitaires ? Quelle diffusion et quels risques de dissémination ? Le COMEPRA, Comité d'éthique et de précaution de l'INRA, s'est prononcé favorablement par rapport aux recherches sur le clonage et les travaux en cours font l'objet d'un encadrement adapté. Dans cet esprit, il faut donc simultanément poursuivre la construction de la position de l'INRA en matière de réflexion éthique et d'acceptabilité sociale (évaluation des attentes sociétales, dialogue avec la société), et engager les recherches nécessaires pour consolider notre expertise scientifique. Des progrès sont attendus dans le domaine de la production de connaissances (mise au point et maîtrise de nouvelles techniques, recherche fondamentale en physiologie et physio-pathologie) qui relève strictement du domaine scientifique, de l'innovation thérapeutique (production de protéines recombinantes d'intérêt médical ou vétérinaire, création et utilisation de modèles animaux de maladies humaines) qui devra être partagé avec nos partenaires du monde médical, ou enfin de l'innovation agronomique (résistance génétique aux maladies, composition des produits animaux), un domaine très prospectif qu'il faudra accompagner au cas par cas et auquel il faudra associer des partenaires économiques.

\section{5 / Les maladies infectieuses animales, émergentes et zoono- tiques}

La pathologie des différentes espèces d'animaux de rente reste dominée par les processus infectieux, émergents ou récurrents. La politique sanitaire vétérinaire de ces dernières années (campagnes de prophylaxie, vaccination systé- 
matique, éradication des éventuels foyers de réintroduction) a eu pour résultat l'élimination spectaculaire de grandes panzooties ce qui a installé une impression générale et erronée de sécurité. Les évènements sanitaires récents (maladies à prions, fièvre aphteuse, SRAS, influenza aviaire, fièvre catarrhale ovine) ont replacé l'émergence et la réémergence des maladies infectieuses des animaux au cœur des nouvelles questions de santé animale et de santé publique vétérinaire. De nombreux facteurs favorables aux émergences se combinent actuellement : plasticité et adaptation des agents pathogènes, perte d'efficacité de nombreuses molécules utilisées, modifications climatiques et écologiques, évolution des systèmes d'élevage, intensification des mouvements de personnes et d'animaux, émergence de grands bassins de production et de consommation en Asie. Par ailleurs, la maitrise des maladies animales et la sécurité des produits qui en résulte, constituent la première priorité des filières animales et des gestionnaires du risque, d'autant que l'évolution réglementaire européenne repousse vers l'amont la responsabilité de la qualité sanitaire des produits. Les entités pathologiques concernées relèvent à la fois des maladies émergentes ou réémergentes mais aussi de maladies endémiques infectieuses et parasitaires qui nécessitent une attention d'autant plus vive que le contexte réglementaire pousse à un usage prudent et raisonné des anti-infectieux, première méthode de contrôle.

Ces nouveaux défis posent de nombreuses questions scientifiques qui relèvent d'approches disciplinaires et intégratives et d'une véritable écologie des maladies infectieuses, intégrant la dimension environnementale. Nos efforts dans ce domaine devront être soutenus et activement coordonnés avec ceux du CIRAD dans le cadre du groupement de recherche qui s'organise autour des maladies émergentes et/ou zoonotiques, y compris les maladies vectorisées par les arthropodes. Cette structuration et cette programmation commune entre nos deux organismes devront être un cadre pour nos travaux futurs, complémentaire de la consolidation de notre partenariat avec les ENV, et un élément moteur pour l'élargissement de nos partenariats nationaux (AFSSA) et internationaux. Plus précisément, plusieurs grands domaines de recherche seront explorés : la génomique structurale et fonctionnelle des agents pathogènes, les aspects génétiques, physiopathologiques et immunologiques de la réponse de l'hôte, la biologie et l'écologie des interactions entre vecteurs, hôte et agents pathogènes, la pharmacologie vétérinaire, l'épidémiologie et la modélisation de la propagation et de la circulation des agents infectieux, les bases de la décision et de la gestion des maladies animales et l'économie de la santé.

Plus généralement, devant les enjeux forts de développement des produits animaux pour répondre à la demande alimentaire du Sud, et en considérant les contributions de plus en plus importantes des pays émergents et en développement aux connaissances biologiques et systémiques, les recherches sur les productions animales en régions chaudes ne seront véritablement efficaces que si une coordination et une programmation commune se met en place entre l'INRA et le CIRAD, à l'image de ce qui se construit en santé animale. Ces travaux devront à la fois intégrer les diverses composantes des systèmes d'élevage (caractéristiques génétiques et physiologiques des animaux, caractérisation et gestion des ressources alimentaires, qualité des produits, nature même des systèmes d'élevage, viabilité économique, gestion de la santé animale, élevage et territoire), et concevoir de nouveaux systèmes permettant de répondre aux enjeux nutritionnels et environnementaux.

\section{Conclusion}

Les recherches en productions animales de demain devront tout à la fois produire des données génériques pour la connaissance du vivant et proposer des systèmes de production innovants et compétitifs, en rupture avec l'existant. Elles devront prendre en compte la dimension mondiale des problématiques et s'accompagner d'une large ouverture disciplinaire et partenariale. Dans cet esprit, un effort particulier devra être fourni sur les relations élevage-environnement et sur l'élaboration et la maîtrise de la qualité des produits animaux. Enfin, elles devront s'inscrire dans le cadre d'un dialogue renouvelé avec les citoyens qui conditionnera la place future de l'animal, de l'élevage et de ses produits dans la société moderne.

\section{Références}

Boiffin J, Hubert B., Durand N., 2004. Agriculture et développement durable. Enjeux et questions de recherche. Publication INRA, $92 \mathrm{p}$.

Chevassus-au-Louis B., 2006. Refonder la recherche agronomique. Les leçons inaugurales du groupe ESA (Ed), 57-101.

Devine R., 2003. La consommation des produits carnés. INRA Prod. Anim., 16, 325-327.

FAO, 2006. Livestock long shadow; environmental issues and options. Food and Agriculture
Organisation of the United Nations, Rome 2006, 390 p.

Guyomard H., Le Mouël C., Jez C., Forslund A., Foumel E., 2007. Prospective Agriculture 2013. Résultats et enseignements principaux par thème. Croissance, environnement, biocarburants, négociations commerciales : une agriculture européenne sous influences multiples et contradictoires; des défis pour la Politique Agricole Commune de demain. www.inra.fr/ agriculture 2013/colloque agriculture 2013
Redlingshöfer B. 2006. Vers une alimentation durable ? Ce qu'enseigne la littérature scientifique. Courrier Env. INRA, 53, 83-102.

Soussana J.F., Führer J., Jones M., Van Amstel, A., 2007. The greenhouse gas balance of grasslands in Europe. Preface. Agric. Ecosyst. Env., 121, 1-4.

Veissier I., Beaumont C., Lévy F., Larrère R., (Coord.), 2007. Le bien-être animal. Numéro spécial, INRA Prod. Anim., 20, 100p. 


\section{Résumé}

La dimension planétaire des problématiques de recherche (développement durable, changement climatique, gestion de la biodiversité, qualité de l'eau, qualité et sécurité de l'alimentation, maladies émergentes, bioénergies), la nécessité d'accroître encore à l'avenir l'offre alimentaire mondiale pour répondre à une démographie galopante, et l'évolution du statut de l'animal replacent les pratiques d'élevage, les animaux et leurs produits au cœur de débats de société. Après un tour d'horizon des grands enjeux auxquels seront confrontés les agronomes de demain, quelques axes de recherche prioritaires sont brièvement esquissés. Ils entrent totalement dans l'objectif d'une recherche agronomique finalisée pour une alimentation adaptée, un environnement préservé et une agriculture compétitive et durable, en lien avec l'ensemble des acteurs publics et privés. Les relations entre l'élevage, ses produits et l'environnement devront être explorées, analysées, modélisées pour faire évoluer nos pratiques et tenter de réconcilier élevage et écologie. L'analyse des grands enjeux qui conditionnent l'évolution de la place des produits animaux dans l'alimentation de l'Homme permettra de revisiter notre dispositif de recherche et d'apporter une dimension intégrative indispensable aux travaux de recherche. Le formidable potentiel de progrès et d'innovation offert par la génomique et la post-génomique devra être exploré. La maîtrise des processus infectieux, émergents ou récurrents, nécessitera la mise œuvre d'une véritable écologie des maladies, intégrant la dimension environnementale. Pour s'approprier pleinement ces différentes dimensions, les chercheurs en sciences animales devront résolument ouvrir leurs réflexions et leurs projets à d'autres disciplines (écologie, agronomie, nutrition humaine, sociologie, économie de l'élevage et des produits...), et construire leurs questions de recherche en diversifiant leurs partenariats, dans le cadre d'un dialogue renouvelé et constructif avec la société.

\section{Abstract}

\section{What future for research in animal production and animal health?}

The planetary dimension of research problems (sustainable development, climatic changes, biodiversity management, water quality, food safety, emerging diseases and bioenergies), the growing need in the future to increase world food supplies as an answer to an increasing demography and the evolution of the animals' status have placed the animal, breeding management, and their products at the centre of the society's debates. After looking at the issues to which tomorrows' agronomist will be confronted, some research axes that have priority are discussed. They are part of the objective for a finalised agronomical research for adapted foods, a preserved environment and a competitive and lasting agriculture and involve both public and private actors. The relation between breeding, its products and the environment must be explored, analysed, modelled in order for practices to evolve and for ecology and farming to be reconciled. The analysis of the big issues that condition the evolution of the place of animal products in human food will allow the revision of our research system and will include an integrative dimension that is essential. The large potential of progress and innovation offered by genomics and post-genomics should be explored. The mastering of infectious processes, emerging or old, necessitates the development of a real ecology of diseases, including environmental aspects. In order to really master these different dimensions, the researcher in animal science will have to open his/her thoughts and projects to those of other fields of study (ecology, agronomy, human nutrition, sociology, farming economics, products...) and to build their research questions by diversifying their partnerships, within the framework of a renewed and constructive dialogue with society.

HERPIN P., CHARLEY B., 2008. Quel avenir pour les recherches en productions et santé animales ? INRA Prod. Anim., 21, 137-144. 
